\title{
Online signature verification using hybrid wavelet transform
}

\author{
Manoj Chavan', Ravish R. Singh ${ }^{2}$, Vinayak Bharadi ${ }^{3}$ \\ ${ }^{1}$ Department of Electronics and Telecommunication Engineering, Thakur College of Engineering and Technology, India \\ ${ }^{2}$ Academic Advisor, Thakur Educational Trust, Mumbai, India \\ ${ }^{3}$ Information Technology Department, Finolex Academy of Management and Technology, India
}

\begin{tabular}{|c|c|}
\hline Article Info & ABSTRACT \\
\hline Article history: & Online signature verification is a prominent behavioral biometric trait. \\
\hline & It offers many dynamic features along with static two dimensional signature \\
\hline Received Dec 22, 2017 & image. In this paper, the Hybrid Wavelet Transform (HWT) was generated \\
\hline Revised Oct 18, 2019 & using Kronecker product of two orthogonal transform such as DCT, DHT, \\
\hline Accepted Oct 30, 2019 & $\begin{array}{l}\text { Haar, Hadamard and Kekre. HWT has the ability to analyze the signal at } \\
\text { global as well as local level like wavelet transform. HWT-1 and }-2 \text { was }\end{array}$ \\
\hline Keywords: & $\begin{array}{l}\text { applied on the first } 128 \text { samples of the pressure parameter and first } 16 \\
\text { samples of the output were used as feature vector for signature verification. }\end{array}$ \\
\hline HMM & This feature vector is given to Left to Right HMM classifier to identify \\
\hline HWT & FAR and FRR. For HWT-2, KEKRE 128 offers best FAR and FRR. HWT-1 \\
\hline Left Right Model & offers better performance than HWT-2 in terms of FAR and FRR. \\
\hline Online Signature Verification & $\begin{array}{l}\text { As the number of states increase, the performance of the system } \\
\text { improves. For HWT-1, KEKRE } 128 \text { offers best performance at } 275 \text { symbols } \\
\text { whereas for HWT-2, best performance is at } 475 \text { symbols by KEKRE } 128 \text {. }\end{array}$ \\
\hline
\end{tabular}

Copyright @ 2020 Institute of Advanced Engineering and Science. All rights reserved.

Corresponding Author:

Manoj Chavan,

Department of Electronics and Telecommunication Engineering,

Thakur College of Engineering and Technology,

Thakur Village, Kandivali East Mumbai-400101 Maharashtra, India.

Email: prof.manoj@gmail.com

\section{INTRODUCTION}

Handwritten Signatures have been used for centuries for identification and authentication of a person as well as documents [1, 2]. In biometrics classification, it is part of behavioral characteristics like voice, gait etc. whereas physical characteristics include fingerprint, palm print, face, iris, retina etc. [3]. Biometric characteristics are universal, unique and measurable and are better than personal ID cards, PIN or passwords [4-6]. Biometric system for signatures can operate in two ways. First Verification, in which the individual's signature will be compared with his stored signature in a database to verify that the individual is the same who he says to be. Second Identification, in which the signature will be compared with the many signatures in the database to identify an individual out of many unknowns.

Automating the process of Handwritten Signature Verification will be useful for document verification in various sectors such as banking, legal documentation etc. There are two types of Signature Verification; offline (static) or online (dynamic). Offline signatures offer two dimensional image of the signatures whereas online signatures have added advantage that it also measures pressure applied by the user, speed of writing, inclination of pen along with the two dimensional signature image [7]. Dr. Kekre proposed Hybrid Wavelet Transform (HWT) which is formed by combining the two orthogonal transforms using Kronecker product. It has the ability to analyze the signal at global as well as local level like wavelet transform [8]. HWT is of two types and are explained below. Consider matrices X and Y as shown below. 


$$
\mathrm{X}=\left[\begin{array}{cccc}
x_{11} & x_{12} & \ldots & x_{1 a} \\
x_{21} & x_{22} & \ldots & x_{2 a} \\
\ldots & \ldots & \ldots & \ldots \\
x_{a 1} & x_{a 2} & \ldots & x_{a a}
\end{array}\right] \quad \mathrm{Y}=\left[\begin{array}{cccc}
y_{11} & y_{12} & \ldots & y_{1 b} \\
y_{21} & y_{22} & \ldots & y_{2 b} \\
\ldots & \ldots & \ldots & \ldots \\
y_{b 1} & y_{b 2} & \ldots & y_{b b}
\end{array}\right]
$$

HWT-1 matrix ' $\mathrm{T}_{\mathrm{XY}}$ ' of size $(\mathrm{NxN})$, as shown in Table 1, can be formed by the Kronecker product of two orthogonal transform matrices $\mathrm{X}$ and $\mathrm{Y}$ respectively, with sizes ( $\mathrm{a} \mathrm{x}$ ) and ( $\mathrm{b} \mathrm{x}$ ), such that $\mathrm{N}=\mathrm{a} \mathrm{x} \mathrm{b}$. For HWT-1, first ' $b$ ' number of rows of the HWT matrix are calculated as the product of each element of first row of the orthogonal transform X with each of the columns of the orthogonal transform Y. For next ' $b$ ' number of rows of HWT matrix the second row of the orthogonal transform matrix $\mathrm{X}$ is shift rotated after being appended with zeros. Similarly the other rows of HWT matrix are generated as set of $b$ rows each time for each of the 'a-1' rows of orthogonal transform matrix X starting from second row up to last row.

Table 1. HWT-1 Matrix

\begin{tabular}{|c|c|c|c|c|c|c|c|c|c|c|c|c|c|}
\hline$y 11 x 11$ & y11x12 & $\ldots$ & y11x1a & $\mathrm{y} 12 \mathrm{x} 11$ & $\mathrm{y} 12 \mathrm{x} 12$ & $\ldots$ & y12x1a & $\ldots$ & $\ldots$ & y1bx 11 & $\mathrm{y} 1 \mathrm{bx} 12$ & $\ldots$ & y1bx $1 \mathrm{a}$ \\
\hline $\mathrm{y} 21 \mathrm{x} 11$ & $\mathrm{y} 21 \mathrm{x} 12$ & $\ldots$ & y21x $1 a$ & $\mathrm{y} 21 \mathrm{x} 12$ & $\mathrm{y} 22 \times 12$ & $\ldots$ & $\mathrm{y} 22 \mathrm{x} 1 \mathrm{a}$ & $\ldots$ & $\ldots$ & $\mathrm{y} 2 \mathrm{~b} \times 11$ & $\mathrm{y} 2 \mathrm{~b} \times 12$ & $\ldots$ & $y 2 b x 1 a$ \\
\hline$\ldots$ & & $\ldots$ & $\ldots$ & $\ldots$ & & $\ldots$ & 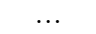 & $\ldots$ & $\ldots$ & 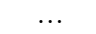 & $\ldots$ & $\ldots$ & $\ldots$ \\
\hline yb1x11 & yb1x12 & $\ldots$ & yb1x11 & $\mathrm{yb} 2 \times 11$ & $y b 2 \times 12$ & $\ldots$ & $\mathrm{yb} 2 \times 1 \mathrm{a}$ & $\ldots$ & $\ldots$ & yb1x 11 & yb1x12 & $\ldots$ & $y b 1 \times 12$ \\
\hline $\mathrm{x} 21$ & $\mathrm{x} 22$ & $\ldots$ & $\mathrm{x} 2 \mathrm{a}$ & 0 & 0 & $\ldots$ & 0 & $\ldots$ & $\ldots$ & 0 & 0 & $\ldots$ & 0 \\
\hline 0 & 0 & $\ldots$ & 0 & $\mathrm{x} 21$ & $\mathrm{x} 22$ & $\ldots$ & $x 2 a$ & $\ldots$ & $\ldots$ & 0 & 0 & $\ldots$ & 0 \\
\hline$\ldots$ & $\ldots$ & $\ldots$ & $\ldots$ & $\ldots$ & $\ldots$ & $\ldots$ & $\ldots$ & $\ldots$ & $\ldots$ & $\ldots$ & $\ldots$ & $\ldots$ & $\ldots$ \\
\hline 0 & 0 & $\ldots$ & 0 & 0 & 0 & $\ldots$ & 0 & $\ldots$ & $\ldots$ & $\mathrm{x} 21$ & $\mathrm{x} 22$ & $\ldots$ & $\mathrm{x} 2 \mathrm{a}$ \\
\hline x31 & x 32 & $\ldots$ & $\mathrm{x} 3 \mathrm{a}$ & 0 & 0 & $\ldots$ & 0 & $\ldots$ & $\ldots$ & 0 & 0 & $\ldots$ & 0 \\
\hline 0 & 0 & $\ldots$ & 0 & x 31 & x 32 & $\ldots$ & $\mathrm{x} 3 \mathrm{a}$ & $\ldots$ & $\ldots$ & 0 & 0 & $\ldots$ & 0 \\
\hline$\ldots$ & $\ldots$ & $\ldots$ & $\ldots$ & $\ldots$ & $\ldots$ & $\ldots$ & $\ldots$ & $\ldots$ & $\ldots$ & $\ldots$ & $\ldots$ & $\ldots$ & $\ldots$ \\
\hline 0 & 0 & $\cdots$ & 0 & 0 & 0 & $\ldots$ & 0 & $\ldots$ & $\ldots$ & $\mathrm{x} 31$ & x32 & $\ldots$ & $\mathrm{x} 3 \mathrm{a}$ \\
\hline$\cdots$ & $\cdots$ & $\cdots$ & $\cdots$ & $\cdots$ & $\cdots$ & $\cdots$ & $\cdots$ & $\cdots$ & $\cdots$ & $\cdots$ & $\cdots$ & $\cdots$ & $\cdots$ \\
\hline$\ldots$ & $\ldots$ & $\cdots$ & $\ldots$ & $\cdots$ & $\cdots$ & $\cdots$ & $\ldots$ & $\ldots$ & $\ldots$ & $\ldots$ & $\ldots$ & $\ldots$ & $\ldots$ \\
\hline xa1 & $\mathrm{xa} 2$ & $\ldots$ & xaa & 0 & 0 & $\ldots$ & 0 & $\ldots$ & $\ldots$ & 0 & 0 & $\ldots$ & 0 \\
\hline 0 & 0 & $\cdots$ & 0 & xa1 & xa2 & $\ldots$ & хаa & $\ldots$ & $\ldots$ & 0 & 0 & $\ldots$ & 0 \\
\hline$\cdots$ & $\ldots$ & $\ldots$ & $\cdots$ & $\cdots$ & $\cdots$ & $\ldots$ & $\ldots$ & $\ldots$ & $\ldots$ & $\cdots$ & $\cdots$ & $\ldots$ & $\ldots$ \\
\hline 0 & 0 & $\ldots$ & 0 & 0 & 0 & $\ldots$ & 0 & $\ldots$ & $\ldots$ & xa1 & $\mathrm{xa} 2$ & $\ldots$ & xaa \\
\hline
\end{tabular}

HWT-2 matrix of size $(\mathrm{NxN})$ is also formed by the Kronecker product of two orthogonal transform matrices $X$ and $Y$. First N/2 rows of the matrix are formed by product of each element of first a/2 rows of the matrix $X$ with each of the columns of the matrix Y. For next ' $b$ ' number of rows of matrix, the ' $a / 2+1$ 'th row of the orthogonal transform matrix $\mathrm{X}$ is shift rotated after being appended with zeros. Next N/2 rows are generated as set of $b$ rows each time for each of the ' $a / 2$ ' rows of orthogonal transform matrix $X$ starting from ' $a / 2+1$ 'th row up to last row.

HWT offers better performance in image compression than the orthogonal transforms used to generate them [9, 10]. HWT is also used for water marking [11] and to convert color image to gray image [12]. Various classifiers based on KNN, SVM and NN [13, 14] have been used for verification of signatures. In [15], KNN classifier was used with, HWTs of the pressure map of online signatures as feature vector. It offered an EER of 30\%. In [16], SVM classifer was used with, a kernel function of online signature time series, based on LCSSs detection, as a feature vector. It offered an EER of $6.84 \%$. Using SVM in conjunction with HMM offered FAR of $1.96 \%$ and FRR of $60.43 \%$. In [17], neural network classifier was used with, the approximation and detail component of DWT of the pen postion and pen movement angle as feature vector. Using all coefficints of DWT, success rate was $100 \%$ with trained signature, $90 \%$ with untrained signatures and FRR of $24 \%$. Using selected 25 coefficients of DWT, success rate was $100 \%$ with trained signature, $95 \%$ with untrained signatures and FAR of $8 \%$.

In this paper, we propose a method for online signature verification using Hybrid Wavelet Transform and Hidden Markov Model classifier. The proposed method is shown in Figure 1. We have used SVC2004 database which is a large database containing signatures from 40 individuals. It has total of 1,600 signatures, obtained using a Wacom Intuos tablet. It consists of 20 genuine and 20 forgery signatures collected for each person. Genuine signatures are collected in two different sessions. Forgeries for each person are provided by at least four other individuals from the database. The performance results of various signature verification systems that participated in the SVC2004 competition is available. The best performance for 40 available users is average EER $6.90 \%$ with standard deviation of $9.45 \%$, minimum value of 0.00 and maximum value of $50.00 \%$. The best performance for 60 other users is average EER $2.89 \%$ with standard deviation of 5.69\%, minimum value of 0.00 and maximum value of $30.00 \%$ [18]. Every signature sample consist of $\mathrm{X}$-coordinate-scaled cursor position along the $\mathrm{x}$-axis, Y-coordinate-scaled cursor position along the $\mathrm{y}$-axis, 
Time stamp-system time at the time of signing, Button status-current button status ( 0 for pen-up and 1 for pen-down), Azimuth-clockwise rotation of cursor about the z-axis, Altitude-angle upward toward the +ve z-axis, Pressure-normal pressure applied by hand. Pressure applied by the tip of the pen on the pressure sensitive pad is used for generating the feature vector. We have used Discrete Cosine transform (DCT), Discrete Hartley transform (DHT), Discrete Walsh transform (DWT) and Discrete Kekre transform (DKT) to form the HWT-1 and HWT-2 matrix. The output of HWT is given to HMM for classification.

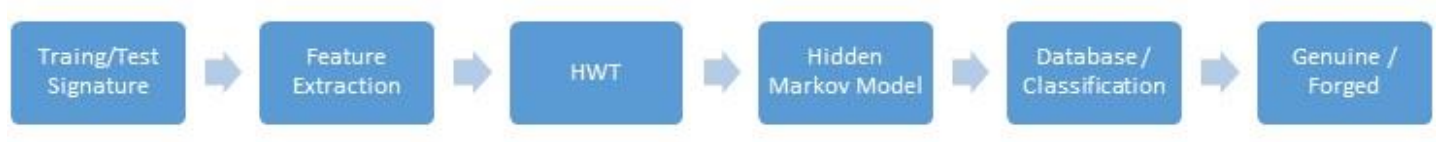

Figure 1. Proposed System

\section{RESEARCH METHOD}

Signature database of 1600 signatures, provided by The First International Signature Verification Competition (SVC 2004) is used. It has signatures of 40 users. Every user has 40 signatures, out of which, 20 are genuine and 20 are skilled forgeries. The first 128 samples of every signature is used to find HWT. The signatures, having samples less than 128, will be padded with zeroes. The first 16 samples of the HWT output are used as feature vector. Discrete Cosine transform (DCT), Discrete Hartley transform (DHT), Discrete Walsh transform (DWT) and Discrete Kekre transform (DKT) are used to form the HWT [19, 20]. There are many topologies of HMM such as Left to Right, Ergodic and Ring. Left to Right topology as shown in Figure 2, is found to be best suited for the Signature modelling [21, 22].

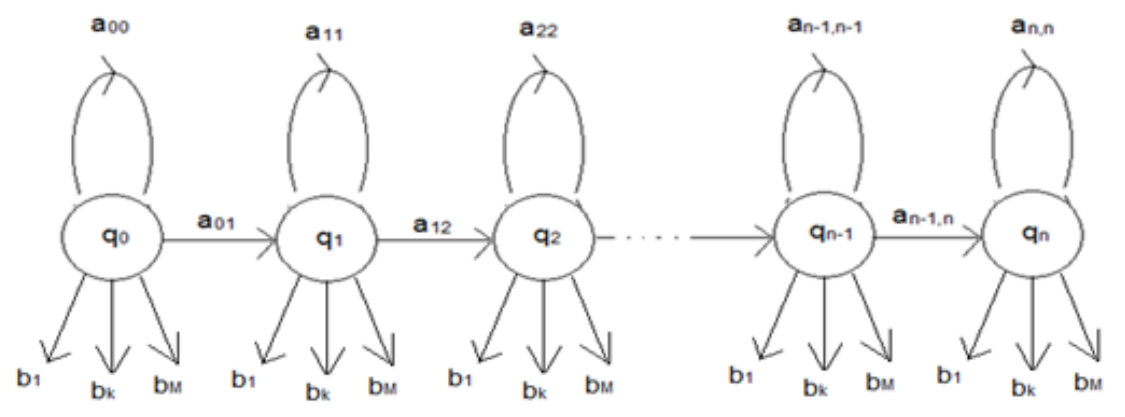

Figure 2. Left to Right HMM model

HMM is represented by the transition probability matrix (A), Observation matrix (B) and initial probability distribution matrix $(\pi)$. $[23,24]$ Consider a system which is in a distinct state $(\mathrm{S} 1, \mathrm{~S} 2, \ldots, \mathrm{SN})$ at any point of time. In this experiment the number of states $(\mathrm{N})$ of the model are varied from 2 to 5 . As the number of states increase, the time needed for training increases. The number of observations (M) corresponding to each state are varied from 200 to 750 in the increments of 25 . The output of HWT is a matrix of dimension [1×128]. The matrix elements from 1 to 16 are chosen as a feature vector. Feature vectors are scaled into $M$ number of observations.

Initial Probability Distribution $(\pi): \pi_{\mathrm{i}}=\mathrm{P}\left(\mathrm{q}_{1}=\mathrm{S}_{\mathrm{i}}\right) ; 1 \leq \mathrm{i} \leq \mathrm{N}$. We assume the initial probability of the first state is 1 and the others are 0 which implies that in the beginning HMM is always in state 1. State transition probability $\left(a_{i j}\right)$ : $a_{i j}=P\left(S_{t}=j / S_{t-1}=i\right)$. For the left-to-right HMM, $a_{i j}=0$ when $i>j$. we are using the HMM of first order so that $a_{i j}=0$ when $j>i+1$. Initially, the state transition matrix is generated using the random numbers such that $\sum_{j=1}^{N}$ aij $=1 ; 1 \leq \mathrm{i} \leq \mathrm{N}$. Observation probability $\left(\mathrm{b}_{\mathrm{j}}\right): \mathrm{b}_{\mathrm{j}}(\mathrm{k})=\mathrm{P}\left(\mathrm{V}_{\mathrm{k}}\right.$ at $\left.\mathrm{t} / \mathrm{q}_{\mathrm{t}}=\mathrm{S}_{\mathrm{j}}\right)$; $1 \leq \mathrm{j} \leq \mathrm{N} ; 1 \leq \mathrm{k} \leq \mathrm{M}$; the probability of generating a symbol $\mathrm{V}_{\mathrm{k}}$ in state $\mathrm{j}$.

Statistics and machine learning toolbox of the MATLAB 13 was used for implementation of HMM. Initially a randomly generated transition probability Matrix (A) is generated using MATLAB. We assume observation probability matrix (B) to have equal probability for every symbols and HMM to be in state 1 . HMM is trained using the function 'hmmtrain' for 3 to 20 genuine training signature samples, number of states from 2 to 5 and symbols from 200 to 750. After HMM is trained, it is used to test 20 genuine and 20 forged signatures of 40 users. 


\section{RESULTS AND DISCUSSION}

Performance of the system will be measured on the basis of False Rejection Ratio (FRR) and False Acceptance Ratio (FAR). FRR refers to false rejection of genuine signature and FAR refers to false acceptance of forged signature [25]. FRR is computed as ratio of the number of signatures detected as forged to the total number of genuine signatures tested. FAR is computed as ratio of the number of signatures detected as genuine to the total forged signatures tested. Testing has been carried out for 40 users and then the average FRR and FAR are calculated. In FRR-FAR plot shown in Figure 3, the point where two graphs cross each other is referred as Equal Error Rate (EER). At this point the value of FRR and FAR is minimum. The results obtained by the first 1-16 samples of HWT-1 and 2 for DCT, DHT, HAAR, HADAMARD and KEKRE combinations is shown in the Tables 2-4.

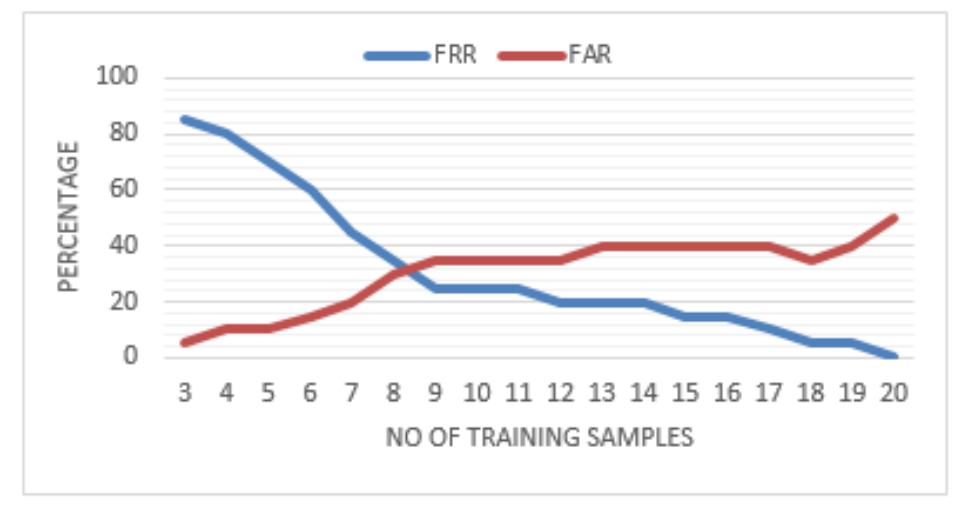

Figure 3. FRR - FAR plot

The comparison of HWT-1 and 2 for 1-16 bit for DCT combinations is shown in the Tables 2-4.

- Best FRR-FAR: FRR-FAR should be as low as possible. For HWT-1, DCT HAAR offers best performance with FRR \& FAR of $0 \%$. For HWT-2, DCT KEKRE offers best performance with FRR \& FAR of $9 \%$. The performance offered by DCT HAAR HWT for HWT-1 is better than HWT-2. The performance offered by DCT combinations for HWT-1 is better than HWT-2. For HWT-1, the performance offered by DCT combinations except DCT DHT is better than Orthogonal DCT transform. For HWT-2, only DCT KEKRE offers better performance than DCT combinations than Orthogonal DCT transform.

- Best Number of Training Samples: The number of training samples should be as low as possible. For HWT-1, DCT DHT offers best performance of 12 compared to 15 training samples for Orthogonal DCT transform. For HWT-2, Orthogonal DCT transform offers best performance of 4 training samples compared to all combinations of DCT HWT. The performance offered by DCT combinations for HWT-2 is better than HWT-1.

- Best state wise FRR-FAR: FRR-FAR should be as low as possible for the given state from 2 to 5. For HWT-1, DCT HAAR offers best performance for 2, 4 and 5 states, DCT KEKRE for state 3 and DCT HAAR for state 5 compared to orthogonal DCT transform. For HWT-2, DCT KEKRE offers best performance for 2 to 5 states compared to orthogonal DCT transform. The performance offered by DCT combinations for HWT-1 is better than HWT-2.

- Best Number of Symbol: It should be as low as possible. Testing was carried out for number of symbols from 200 to 750. It evident that the best performance in terms of FRR-FAR, AAR-ARR, EER is offered by 275-325 symbols for HWT-1 and 450-500 symbol for HWT-2. The performance offered by DCT combinations for HWT-1 is better than HWT-2.

The comparison of HWT-1 and 2 for 1-16 bit for DHT combinations is shown in the Table 2-4.

- Best Number of Training Samples: For HWT-1, DHT KEKRE offers best performance with FRR \& FAR of $5 \%$. For HWT-2, DHT KEKRE offers best performance with FRR 13\% \& FAR of $19 \%$. The performance offered by DHT KEKRE HWT for HWT-1 is better than HWT-2. The performance offered by DHT combinations for HWT-1 is better than HWT-2. For HWT-1, the performance offered by DHT DCT, DHT KEKRE is better than Orthogonal DHT transform. For HWT-2, only DHT KEKRE offers better performance than Orthogonal DHT transform. 
- Best Number of Training Samples: For HWT-1, DHT HADAMARD offers best performance of 10 training samples compared to 13 training samples for Orthogonal DHT transform. For HWT-2, DHT HADAMARD offers best performance of 5 training samples compared to 7 training samples for Orthogonal DHT transform. DHT combinations offer better performance for HWT-2 than HWT - 1.

- Best state wise FRR-FAR: For HWT-1, DHT KEKRE offers best performance for 2, 3 and 4 states and DHT DCT for state 5 compared to orthogonal DHT transform. For HWT-2, DHT KEKRE offers best performance for 2 to 5 states compared to orthogonal DHT transform. DHT combinations offer better performance for HWT-1 than HWT-2.

- Best Number of Symbol: Testing was carried out for number of symbols from 200 to 750. It evident that the best performance in terms of FRR-FAR, AAR-ARR, EER is offered by 275 symbols for HWT-1 and 450-500 symbols for HWT-2. DHT combinations offers better performance for HWT-1 than HWT-2.

The comparison of HWT-1 and 2 for 1-16 bit for HAAR combinations is shown in the Tables 2-4.

- Best FRR-FAR:For HWT-1, HAAR DCT and HAAR KEKRE offers best performance with FRR \& FAR of $0 \%$. For HWT-2, HAAR KEKRE offers best performance with FRR 11\% \& FAR of $12 \%$. The performance offered by HAAR DCT and HAAR KEKRE HWT for HWT-1 is better than HWT-2. The performance offered by HAAR combinations for HWT-1 is better than HWT-2. For HWT-1, the performance offered by all combinations of HAAR is better than Orthogonal HAAR transform. For HWT-2, HAAR DHT and HAAR KEKRE offers better performance than Orthogonal HAAR transform.

- Best Number of Training Samples: For HWT-1, HAAR DHT offers best performance of 12 training samples with FRR, FAR of 15\%, 15\% respectively compared to 13 training samples with FRR, FAR of 10\%, 30\% respectively for Orthogonal DHT transform. For HWT-2, HAAR HADAMARD offers best performance of 5 training samples compared to 6 training samples for Orthogonal HAAR transform. HAAR combinations offer better performance for HWT-2 than HWT-1.

- Best state wise FRR-FAR: For HWT-1, HAAR KEKRE offers best performance for 2 to 5 states. HAAR DCT offers best performance for state 5 compared to orthogonal HAAR transform. For HWT-2, HAAR KEKRE offers best performance for 2 to 5 states compared to orthogonal HAAR transform. HAAR combinations offer better performance for HWT-1 than HWT-2.

- Best Number of Symbol: Testing was carried out for number of symbols from 200 to 750. It evident that the best performance in terms of FRR-FAR, AAR-ARR, EER is offered by 275 symbols for HWT-1 and 450-500 symbols for HWT-2. HAAR combinations offer better performance for HWT-1 than HWT-2.

the Table 2-4

The comparison of HWT-1 and 2 for 1-16 bit for HADAMARD combinations is shown in

- Best FRR-FAR: For HWT-1, HADAMARD KEKRE offers best performance with FRR \& FAR of $0 \%$. For HWT-2, HADAMARD KEKRE offers best performance with FRR $25 \%$ \& FAR of $22 \%$. The performance offered by HADAMARD KEKRE HWT for HWT-1 is better than HWT-2. The performance offered by HADAMARD combinations for HWT-1 is better than HWT-2. For HWT-1, the performance offered by all combinations of HADAMARD except HADAMARD DCT is better than Orthogonal HADAMARD transform. For HWT-2, the performance offered by all combinations of HADAMARD is better than Orthogonal HADAMARD transform

- Best Number of Training Samples: For HWT-1, Orthogonal HADAMARD transform offers best performance of 8 training samples compared to all combinations of HADAMARD HWT. For HWT-2, Orthogonal HADAMARD transform offers best performance of 5 training samples compared to all combinations of HADAMARD HWT. HADAMARD combinations offer better performance for HWT-2 than HWT-1.

- Best state wise FRR-FAR: For HWT-1, HADAMARD KEKRE offers best performance for 2 and 5 states. HADAMARD DHT offers best performance for state 4 and 5 compared to orthogonal HADAMARD transform. For HWT-2, HADAMARD KEKRE offers best performance for 2 to 5 states compared to orthogonal HADAMARD transform. HADAMARD combinations offer better performance for HWT-1 than HWT-2.

- Best Number of Symbol :Testing was carried out for number of symbols from 200 to 750 . It evident that the best performance in terms of FRR-FAR, AAR-ARR, EER is offered by 275-300 symbols for HWT-1 and 500 symbols for HWT-2. HADAMARD combinations offer better performance for HWT-1 than HWT-2. 
The comparison of HWT-1 and 2 for 1-16 bit for KEKRE combinations is shown in the Tables 2-4.

- Best FRR-FAR: For HWT-1, KEKRE DCT, KEKRE HAAR and KEKRE128 offers best performance with FRR \& FAR of $0 \%$. For HWT-2, KEKRE 128 offers best performance with FRR 5\% \& FAR of $2 \%$. The performance offered by KEKRE DCT, KEKRE HAAR and KEKRE128 HWT for HWT-1 is better than HWT-2. The performance offered by KEKRE combinations for HWT-1 is better than HWT-2. For HWT-1, the performance offered by KEKRE 128 is better than all combinations of KEKRE HWT. For HWT-2, the performance offered by KEKRE 128 is better than all combinations of KEKRE HWT.

- Best Number of Training Samples: For HWT-1, KEKRE DHT offers best performance of 11 training samples compared to 20 training samples for Orthogonal KEKRE transform. For HWT-2, KEKRE HADAMARD offers best performance of 6 training samples compared to 16 training samples for Orthogonal KEKRE transform. KEKRE combinations offer better performance for HWT-2 is better than HWT-1.

- Best state wise FRR-FAR: For HWT-1, KEKRE 128 offers best performance for 2 TO 5 states compared to combinations of KEKRE HWT. For HWT-2, KEKRE 128 offers best performance for 2 TO 5 states compared to combinations of KEKRE HWT. KEKRE combinations offer better performance for HWT-1 is better than HWT-2.

- Best Number of Symbol: Testing was carried out for number of symbols from 200 to 750 . It evident that the best performance in terms of FRR-FAR, AAR-ARR, EER is offered by 275 symbols for HWT-1 and 450-500 symbols for HWT-2. KEKRE combinations offer better performance for HWT-1 is better than HWT-2.

Table 2. Best FRR FAR for HWT-1 and 2

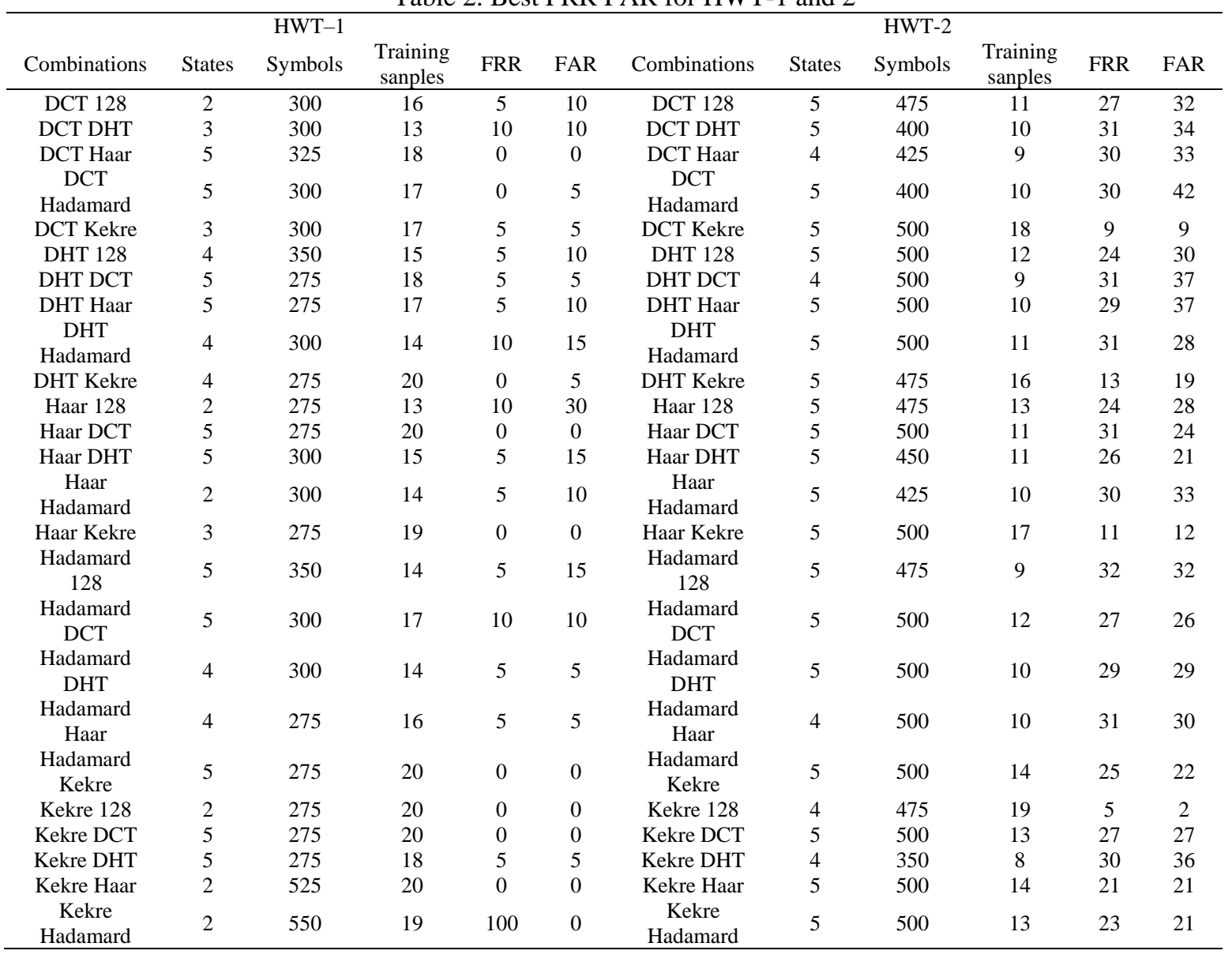


Table 3. Best No. of training samples for HWT-1 and 2

\begin{tabular}{|c|c|c|c|c|c|c|c|c|c|c|c|}
\hline \multicolumn{6}{|c|}{ HWT-1 } & \multicolumn{6}{|c|}{ HWT-2 } \\
\hline Combinations & States & Symbols & $\begin{array}{l}\text { Training } \\
\text { sanples }\end{array}$ & FRR & FAR & Combinations & States & Symbols & $\begin{array}{l}\text { Training } \\
\text { sanples }\end{array}$ & FRR & FAR \\
\hline DCT 128 & 3 & 300 & 15 & 15 & 15 & DCT 128 & 2 & 275 & 4 & 35 & 63 \\
\hline DCT DHT & 2 & 300 & 12 & 15 & 25 & DCT DHT & 3 & 325 & 7 & 33 & 50 \\
\hline DCT Haar & 3 & 300 & 13 & 5 & 20 & DCT Haar & 3 & 375 & 6 & 33 & 47 \\
\hline $\begin{array}{c}\text { DCT } \\
\text { Hadamard }\end{array}$ & 2 & 275 & 12 & 30 & 30 & $\begin{array}{c}\text { DCT } \\
\text { Hadamard }\end{array}$ & 4 & 325 & 6 & 30 & 51 \\
\hline DCT Kekre & 3 & 300 & 17 & 5 & 5 & DCT Kekre & 2 & 450 & 11 & 22 & 30 \\
\hline DHT 128 & 2 & 350 & 13 & 10 & 20 & DHT 128 & 2 & 375 & 7 & 32 & 40 \\
\hline DHT DCT & 4 & 275 & 15 & 5 & 10 & DHT DCT & 2 & 500 & 8 & 33 & 43 \\
\hline DHT Haar & 2 & 275 & 13 & 20 & 20 & DHT Haar & 2 & 350 & 6 & 34 & 46 \\
\hline $\begin{array}{c}\text { DHT } \\
\text { Hadamard }\end{array}$ & 2 & 300 & 10 & 20 & 25 & Dht Hadamard & 4 & 275 & 5 & 29 & 52 \\
\hline DHT Kekre & 2 & 275 & 19 & 5 & 5 & DHT Kekre & 2 & 475 & 12 & 23 & 27 \\
\hline Haar 128 & 2 & 275 & 13 & 10 & 30 & Haar 128 & 2 & 325 & 6 & 32 & 57 \\
\hline Haar DCT & 2 & 275 & 13 & 15 & 0 & Haar DCT & 4 & 350 & 7 & 32 & 52 \\
\hline Haar DHT & 3 & 275 & 12 & 15 & 15 & Haar DHT & 2 & 375 & 6 & 37 & 49 \\
\hline $\begin{array}{c}\text { Haar } \\
\text { Hadamard }\end{array}$ & 3 & 275 & 13 & 10 & 20 & $\begin{array}{c}\text { Haar } \\
\text { Hadamard }\end{array}$ & 3 & 275 & 5 & 32 & 57 \\
\hline Haar Kekre & 3 & 275 & 19 & 0 & 0 & Haar Kekre & 3 & 400 & 12 & 20 & 33 \\
\hline Hadamard 128 & 2 & 275 & 8 & 35 & 30 & Hadamard 128 & 2 & 475 & 5 & 38 & 52 \\
\hline $\begin{array}{c}\text { Hadamard } \\
\text { DCT }\end{array}$ & 2 & 275 & 11 & 20 & 15 & $\begin{array}{l}\text { Hadamard } \\
\text { DCT }\end{array}$ & 2 & 375 & 6 & 30 & 45 \\
\hline $\begin{array}{l}\text { Hadamard } \\
\text { DHT }\end{array}$ & 2 & 275 & 11 & 15 & 20 & $\begin{array}{l}\text { Hadamard } \\
\text { DHT }\end{array}$ & 3 & 300 & 6 & 31 & 49 \\
\hline $\begin{array}{l}\text { Hadamard } \\
\text { Haar }\end{array}$ & 2 & 275 & 14 & 5 & 15 & $\begin{array}{l}\text { Hadamard } \\
\text { Haar }\end{array}$ & 2 & 425 & 8 & 34 & 37 \\
\hline $\begin{array}{l}\text { Hadamard } \\
\text { Kekre }\end{array}$ & 2 & 275 & 18 & 10 & 10 & $\begin{array}{l}\text { Hadamard } \\
\text { Kekre }\end{array}$ & 2 & 500 & 11 & 34 & 31 \\
\hline Kekre 128 & 2 & 275 & 20 & 0 & 0 & Kekre 128 & 2 & 450 & 16 & 11 & 16 \\
\hline Kekre DCT & 2 & 275 & 13 & 10 & 15 & Kekre DCT & 2 & 425 & 9 & 31 & 37 \\
\hline Kekre DHT & 2 & 275 & 11 & 15 & 25 & Kekre DHT & 4 & 350 & 8 & 30 & 36 \\
\hline Kekre Haar & 2 & 525 & 20 & 0 & 0 & Kekre Haar & 2 & 500 & 10 & 33 & 38 \\
\hline $\begin{array}{c}\text { Kekre } \\
\text { Hadamard }\end{array}$ & 2 & 550 & 19 & 100 & 0 & $\begin{array}{c}\text { Kekre } \\
\text { Hadamard }\end{array}$ & 2 & 375 & 6 & 34 & 49 \\
\hline
\end{tabular}

Table 4. Best Statewise FRR FAR for HWT-1 and 2

\begin{tabular}{|c|c|c|c|c|c|c|c|c|c|c|c|}
\hline \multicolumn{6}{|c|}{ HWT-1 } & \multicolumn{6}{|c|}{ HWT-2 } \\
\hline Combinations & States & Symbols & $\begin{array}{l}\text { Training } \\
\text { sanples }\end{array}$ & FRR & FAR & Combinations & States & Symbols & $\begin{array}{l}\text { Training } \\
\text { sanples }\end{array}$ & FRR & FAR \\
\hline DCT Haar & 2 & 300 & 14 & 5 & 10 & DCT Kekre & 2 & 450 & 11 & 22 & 30 \\
\hline DCT Kekre & 3 & 300 & 17 & 5 & 5 & DCT Kekre & 3 & 450 & 13 & 19 & 20 \\
\hline DCT Haar & 4 & 275 & 15 & 10 & 10 & DCT Kekre & 4 & 450 & 15 & 17 & 18 \\
\hline DCT Haar & 5 & 325 & 18 & 0 & 0 & DCT Kekre & 5 & 500 & 18 & 9 & 9 \\
\hline DHT Kekre & 2 & 275 & 19 & 5 & 5 & DHT Kekre & 2 & 475 & 12 & 23 & 27 \\
\hline DHT Kekre & 3 & 275 & 19 & 5 & 5 & DHT Kekre & 3 & 500 & 13 & 22 & 20 \\
\hline DHT Kekre & 4 & 275 & 20 & 0 & 5 & DHT Kekre & 4 & 450 & 15 & 15 & 14 \\
\hline DHT DCT & 5 & 275 & 18 & 5 & 5 & DHT Kekre & 5 & 475 & 16 & 13 & 19 \\
\hline Haar Kekre & 2 & 275 & 19 & 5 & 5 & Haar Kekre & 2 & 450 & 12 & 21 & 35 \\
\hline Haar Kekre & 3 & 275 & 19 & 0 & 0 & Haar Kekre & 3 & 400 & 12 & 20 & 33 \\
\hline Haar Kekre & 4 & 275 & 20 & 0 & 5 & Haar Kekre & 4 & 475 & 16 & 14 & 22 \\
\hline Haar Kekre & 5 & 275 & 20 & 0 & 0 & Haar Kekre & 5 & 500 & 17 & 11 & 12 \\
\hline $\begin{array}{l}\text { Hadamard } \\
\text { Kekre }\end{array}$ & 2 & 275 & 18 & 10 & 10 & $\begin{array}{l}\text { Hadamard } \\
\text { Kekre }\end{array}$ & 2 & 500 & 11 & 34 & 31 \\
\hline $\begin{array}{l}\text { Hadamard } \\
\text { DHT }\end{array}$ & 3 & 275 & 12 & 10 & 10 & $\begin{array}{l}\text { Hadamard } \\
\text { Kekre }\end{array}$ & 3 & 500 & 15 & 16 & 44 \\
\hline $\begin{array}{l}\text { Hadamard } \\
\text { DHT }\end{array}$ & 4 & 300 & 14 & 5 & 5 & $\begin{array}{l}\text { Hadamard } \\
\text { Kekre }\end{array}$ & 4 & 500 & 13 & 25 & 27 \\
\hline $\begin{array}{l}\text { Hadamard } \\
\text { Kekre }\end{array}$ & 5 & 275 & 20 & 0 & 0 & $\begin{array}{l}\text { Hadamard } \\
\text { Kekre }\end{array}$ & 5 & 500 & 14 & 25 & 22 \\
\hline Kekre 128 & 2 & 275 & 20 & 0 & 0 & Kekre 128 & 2 & 450 & 16 & 11 & 16 \\
\hline Kekre 128 & 3 & 275 & 20 & 0 & 0 & Kekre 128 & 3 & 500 & 17 & 10 & 11 \\
\hline Kekre 128 & 4 & 275 & 20 & 0 & 0 & Kekre 128 & 4 & 475 & 19 & 5 & 2 \\
\hline Kekre 128 & 5 & 275 & 20 & 0 & 0 & Kekre 128 & 5 & 475 & 19 & 5 & 2 \\
\hline
\end{tabular}


From Tables 2-4, we get following important results.

- FRR-FAR: For HWT-1, DCT HAAR offers best performance with FRR and FAR of $0 \%$. For HWT-2, KEKRE 128 offers best performance of FRR $5 \%$ and FAR $2 \%$. HWT-1 offers better performance than HWT-2.

- Number of training samples: For HWT-1, Orthogonal HADAMARD transform offers best performance of 8 training. For HWT-2, Orthogonal DCT transform offers best performance of 4 training samples. HWT-2 offers better performance than HWT-1.

- State wise FRR-FAR: For HWT-1, KEKRE 128 offers best performance for 2 to 5 states. For HWT-2, KEKRE 128 offers best performance for 2 to 5 states. HWT-1 offers better performance than HWT-2. As the number of states increase, the performance of the system improves. HWT-1 found to offer better performance for 3 to 5 states and HWT-2 for 5 states.

- Number of Symbol: For HWT-1, KEKRE 128 offers best performance at 275 symbols whereas for HWT-2, best performance is at 475 symbols by KEKRE 128.

The proposed system is compared with existing systems in Table 5. The proposed system offers better performance than the existing sytems.

Table 5. Comparision of proposed system with existing systems

\begin{tabular}{ccc}
\hline Paper & FAR $(\%)$ & FRR $(\%)$ \\
\hline$[13]$ & 30 & 30 \\
{$[14]$} & 1.96 & 60.93 \\
{$[15]$} & 0 & 8 \\
Proposed system & 0 & 0 \\
\hline
\end{tabular}

\section{CONCLUSION}

In the proposed system for online signature verification with pressure as feature vector, HWT-1 offers better performance than HWT-2 for various combinations of DCT, DHT, Haar and Hadamard orthogonal transform. But Kekre transform offers better performance than its various combination of HWT-1 and HWT-2. Comparing KNN, SVM and NN classifier with various dynamic parameters as feature vector, HMM offers better performance. This findings show that the HWT with HMM has been a feasible method for feature vector extracton of online signature vector based biometric systems.

\section{REFERENCES}

[1] R. Plamondon, "A kinematic theory of rapid human movements: Part I: Movement representation and generation," Biological Cybernetics, vol. 72, no. 4, pp. 295-307, 1995.

[2] R. Plamondon, "A kinematic theory of rapid human movements: Part II: Movement time and control," Biological Cybernetics, vol. 72, no. 4, pp. 309-320, 1995.

[3] A. K. Jain, A. Ross and S. Prabhakar, "An Introduction to Biometric Recognition," IEEE Transactions on Circuits and Systems for Video Technology, vol. 14, no. 1, p 4-20, Jan 2004.

[4] K. Veeramacheneni, L. A. Osadciw and P. K. Varshney, "An adaptive multimodal biometric management algorithm," IEEE Trans. Systems, Man and Cybernetics. Part C, vol. 35, no. 3, p. 344-356, Aug 2005.

[5] K. Huang and H. Yan, "Signature verification using fractal transformation," Proc. 15th Int. Conf. Pattern Recog. (ICPR-15), Barcelona, Spain, Sept 2000.

[6] S. Nanavati, M. Thieme, and R. Nanavati, "Biometrics: Identity Verification in a Networked World," New York: Wiley, p 123-131, 2002.

[7] T. Ohishi, Y. Komiya, H. Morita, and T. Matsumoto, "Pen-input online signature verification with position, pressure, inclination trajectories," in Proc. 15th Int. Parallel Distrib. Process. Symp. (IPDPS-15), San Francisco, CA, p. 170, Apr. 2001.

[8] H. B. Kekre, T. K. Sarode and S. D. Thepade, "Inception of HWT using Two Orthogonal Transforms and It's use for Image Compression," (IJCSIS) International Journal of Computer Science and Information Security, vol. 9, no. 6, p 80-87, Jun 2011.

[9] H. Kekre, T. Sarode and P. Natu, "Colour Image Compression using DKT-DCT Hybrid Wavelet Transform in Various Colour Spaces," International Journal of Signal Processing, Image Processing and Pattern Recognition, vol. 7, no. 5, pp. 105-124, 2014.

[10] H. Kekre, T. Sarode and N. Prachi, "Image Compression Using Real Fourier Transform, Its Wavelet Transform and Hybrid Wavelet with DCT," International Journal of Advance Computer Science and Application, vol. 4, no. 5, 2013.

[11] H. Kekre, T. Sarode and S. Natu, "Robust Watermarking Technique using Hybrid Wavelet Transform Generated from Kekre Transform and Discrete Cosine Transform," International Journal of Scientific and Research Publications, vol. 4, no. 2, Feb 2014. 
[12] G. B. Atkar and S. Gore, "Enhanced Performance of Color To Gray and Back by Using Hybrid Wavelet Transforms," International Journal of Emerging Technology and Advanced Engineering, vol. 3, no. 4, April 2013.

[13] M. Singhal, M. Trikha, M. Dutta, "Time Independent Signature Verification using Normalized Weighted Coefficients," International Journal of Electrical and Computer Engineering (IJECE), vol. 6, no. 6, p 2658-2664, December 2016.

[14] M. Singhal, M. Trikha, M. Dutta, "Signature Verification using Normalized Static Features and Neural Network Classification," International Journal of Electrical and Computer Engineering (IJECE), vol. 6, no. 6, p 2665-2673, December 2016.

[15] V. A. Bharadi, V. I. Singh and Bhushan Nemade, "Hybrid Wavelets based Feature Vector Generation from Multidimensional Data set for On-line Handwritten Signature Recognition" Proc. 5th Int. Conf. Confluence The next Generation Information Technology Sumit (Confluence) Noida, India, Nov 2014.

[16] C. Gruber, T. Gruber, S. Krinninger, and B. Sick, "Online Signature Verification With Support Vector Machines Based on LCSS Kernel Functions," IEEE Transactions on Systems, MAN. And Cybernatics-Part B: Cybernetics, vol. 40, no. 4, p 1088-1100, Aug 2010.

[17] Maged M.M. Fahmy," Online handwritten signature verification system based on DWT features extraction and neural network classification," Ain Shams Engineering Journal, vol. 1, no. 1, p 59-70, Sept 2010.

[18] SVC2004 Home. http://www.cse.ust.hk/svc2004/. Accessed 02 Dec 2011.

[19] M. Chavan, R. R. Singh and V. A. Bharadi, "Online Signature Verification using HWT with Hidden Markov Model" in Proc 4th International Conference on Computing, Communication, Control And Automation (ICCUBEA), Pune, India Aug 2017.

[20] M. Chavan, R. R. Singh and V. A. Bharadi, "Handwritten Signature Verification using Hidden Markov Model with HWT" in Proc 4th International Conference on Computing, Communication, Control And Automation (ICCUBEA), Pune, India Aug 2017.

[21] S. Garcia-Salicetti and B. Dorizzi, "On using the Viterbi path along with HMM likelihood information for online signature verification," IEEE Trans. Syst., Man, Cybern. B, vol. 37, no. 5, p 1237-1247, Oct 2007.

[22] L. Rabiner, "A tutorial on hidden Markov models and selected applications in speech recognition," Proceedings of IEEE, vol. 77, no. 2, p 257-286, Feb 1989.

[23] M. M. Shafiei and H. R. Rabiee, " A New On-line Verification Algorithm Using Variable length Segmentation and Hidden Markov Models," in Proc 7 th International Conference on Document Analysis and Recognition (ICDAR'03), Singapore, 2003.

[24] H. S. Yoon, J. Y. Lee and H. S. Yang, "An on-line signature verification system using hidden Markov model in polar space," in Proc. 8th Int. Workshop Front. Handwriting Recognit. (IWFHR-8), Ontario, Canada, Aug. 2002.

[25] D. Impedovo and G. Pirlo, "Automatic Signature Verification: The State of the Art," IEEE Transaction on Systems, MAN and Cybernatics Part C: Application and Reviews, vol. 28, no.5, Sept 2008.

\section{BIBLIOGRAPHIES OF AUTHORS}

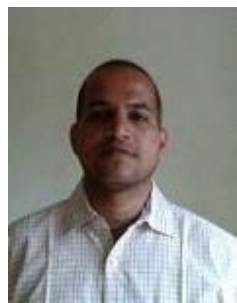

Manoj Chavan received the B.E. degree from the Vidyavardhini's College of Engineering and Technology, University of Mumbai, Maharashtra, India, in 2001, the M.E. degree from the Shivaji University, Kolhapur, India, in 2012, and currently he is doing Ph.D. from Pacific University, Udaipur, India. From 2001 to 2005, He worked as lecturer in the department of Electronics and Telecommunication Engineering at St. Francis Institute of Technology, Borivali, Mumbai-400103, India. He has been working as Assistant Professor in Thakur College of Engineering and Technology, Mumbai, since 2005. He is life member of ISTE, India. His area of interest is Pattern recognition and Machine Learning.

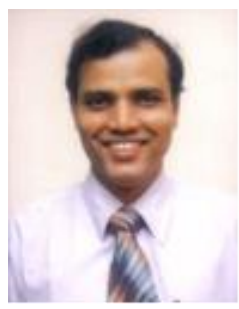

Dr. Ravish R. Singh received B.E. from K.J. Somaiya College of Engineering, Mumbai, University of Mumbai, July 1991 and M. Tech. (Communication Engineering), I.I.T. Bombay, January 2001. He did his Ph.D. (Technology), from Sardar Patel College of Engineering, Mumbai, University of Mumbai, November 2013. He worked as Associate Professor in Electronics and Telecommunication in Thakur College of Engineering and Technology from 1st January, 2006 to 4th July, 2014.He also worked as Assistant Professor in Electronics and Telecommunication Engineering from 20th July, 2002 to 31st December 2005 and as Senior Lecturer in Electronics and Telecommunication Engineering from 8th August, 2001 to 19th July 2002. Now he is holding chair of Academic Advisor at Thakur Educational Trust, Mumbai. He has authored and co-authored several books including Electrical Engineering (McGraw Hill Education, India, 2016), Engineering Mathematics (McGraw Hill Education, India, 2016) Numerical and Statistical Methods for Computer Engineering (McGraw Hill Education, India, 2016), Complex Variables and Numerical Methods (McGraw Hill Education, India, 2015), and Linear Algebra and Vector Calculus (McGraw Hill Education, India, 2014). He is life member of IETE and ISTE, India. 
Dr. Vinayak Ashok Bharadi received the B.E. degree in Electronics from Finolex Academy of Management and Technology, University of Mumbai, in 2002. M.E. degree in Electronics and Telecommunication in 2007 and $\mathrm{PhD}$ in engineering in 2011 from NMIMS University, Mumbai, India. Presently he is working as Associate Professor with Department of Information Technology of Finolex Academy of Management and Technology He is life member of ISTE and area of interest are cloud computing, big data analytics, biometrics and DSP. 